\title{
FUNCTIONAL PEARLS Maximum Marking Problems
}

\author{
Richard S. Bird \\ Programming Research Group, Oxford University \\ Wolfson Building, Parks Road, Oxford, OX1 3QD, UK
}

\section{Introduction}

Here are two puzzles for you to solve. First, consider the binary tree in Figure 1. Take a pencil (I am assuming that this is your personal copy of JFP!) and mark some of the nodes in such a way that the sum of the values of marked nodes is as large as possible. The catch is that you cannot mark all the nodes: if you mark a node, then you are not allowed to mark its parent. Equivalently, no two marked nodes can be contiguous in the tree.

The second puzzle is similar though both the datatype and constraint are different. Consider the rose tree of Figure 2 (a rose tree is a tree with arbitrary branching structure). Mark some of the nodes so that all marked nodes are now contiguous in the tree. For example, if you mark the root value 4 and the leaf value 1, then you must also mark the values 5 and -3 along the path from 4 to 1 . Again the idea is to maximise the sum of the marked nodes. Of course, if all values were nonnegative, the best solution would be to mark all nodes. But they aren't and the maximum sum is obtained only by a judicious choice of marking. Answers to both puzzles are given at the end of the paper.

The maximum marking problem, MMP for short, is the problem of marking the entries of some given data structure in such a way that a given constraint is satisfied and the sum of the values associated with marked entries is as large as possible. By the end of this pearl you will be convinced that there is a linear-time solution for both the puzzles described above.

Other variations of the MMP correspond to some well-known problems. If the

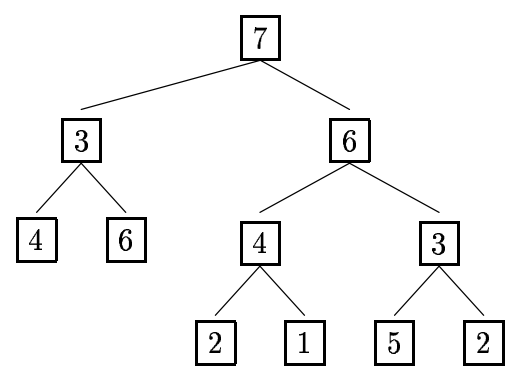

Fig. 1. A binary tree 


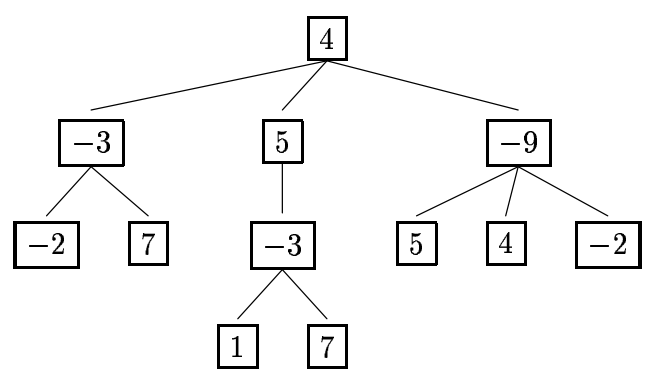

Fig. 2. A rose tree

data structure is a list of items along with their weights and values, and the marking constraint is that the sum of the weights of the items does not exceed a given quantity, then the problem of identifying the maximum value sum is just the classic Knapsack Problem. If the data structure is a list of numbers, and the constraint is that marked entries should be adjacent, then we have the well-known Maximum Segment Sum problem.

The theory behind these problems and how they can be solved efficiently is given in the chapter entitled "Thinning Algorithms" of (Bird and de Moor,1996). The real purpose of this pearl is to try and explain the essential ideas behind thinning algorithms in the context of a specific class of examples, without delving too much into the categorical theory of relations that forms the basis of (Bird and de Moor,1996). This pearl has also been written to try and answer the criticism made in (Sasano et al.,2000) to the effect that thinning algorithms are too difficult for functional progammers to apply in practice.

Let us end this introduction with one way of specifying the marking constraint of the first puzzle. We will mark a tree of type Tree Int, where

$$
\text { data Tree } a=\text { Leaf } a \mid \text { Node } a(\text { Tree } a)(\text { Tree } a)
$$

by attaching a Boolean label to each integer, where a True label indicates that an integer is marked and so contributes to the sum, while a False label indicates that it does not. The constraint is that the binary tree should be atiguously marked, a fancy name for a non-contiguous marking. One way to formalise atiguousness is to say that a marked tree $x$ is atiguous if atig $x$ returns a well-defined value, where

$$
\begin{array}{lll}
\text { atig } & :: & \text { Tree }(\text { Int } \times \text { Bool }) \rightarrow \text { Bool } \\
\text { atig } & = & \text { foldTree base step } \\
\text { base }(n, b) & = & b
\end{array}
$$

and

$$
\text { step }(n, b) \text { bx by }=\left\{\begin{array}{lll}
b \wedge \neg b x \wedge \neg b y & \rightarrow & \text { True } \\
\neg b & \rightarrow & \text { False }
\end{array}\right.
$$

Thus, a tree $x$ is atiguous iff atig $x$ returns either True or False. The function foldTree is the fold function for the type Tree $a$. It is easy to see that atig $x$ is undefined if, during the folding process and evaluation of step on tree $x$, a node is 
encountered which is labelled True and one or both of its daughter nodes is also labelled True. Thus atig is a partial function. Haskell programmers can implement atig as a total function using Maybe Bool as the target type. The important point is that the target type of atig is finite. As we will see, the fact that atig can be expressed as a fold returning a value in a finite set is enough to guarantee a lineartime solution for the problem.

\section{Specification}

Forget binary trees, rose trees or lists, and imagine only that we are given a datatype $T$ Int of integers. The marking problem for $T$ is solved by a function $m m p:: T$ Int $\rightarrow$ Int that returns the maximum sum available. For simplicity we will concentrate only on the value of the best marking rather than on the marking itself, but it is just as easy to consider instead a function $m m p:: T$ Int $\rightarrow T($ Int $\times$ Bool $)$ that returns the best marking.

The function $m m p$ can be specified in the following way:

$$
m m p=\max (\leq) \cdot \Lambda\left(\text { value } \cdot \text { dom test } \cdot \text { map }_{T} \text { mark }\right)
$$

The remainder of this section is devoted to explaining the notation and the subsidiary functions appearing in the specification of $m m p$.

First of all, the specification and its components are interpreted not as Haskell (or ML) functions over types living in the universe $C P O_{\perp}$ of complete partial orders with bottom element $\perp$, but as multifunctions over types in the universe $S E T$ of ordinary sets. A multifunction is a nondeterministic function or, more simply, a relation that associates zero or more results with each argument. We indicate a multifunction by writing its type as $A \leadsto B$ rather than $A \rightarrow B$. For example,

$$
\begin{array}{lll}
\text { mark } & :: \quad \text { Int } \sim \text { Int } \times \text { Bool } \\
\text { mark } n & =(n, \text { True }) \square(n, \text { False })
\end{array}
$$

The box $\square$ signifies nondeterministic choice. Thus mark is a multifunction that attaches an arbitrary Boolean value to an integer. To simplify subsequent type expressions, we introduce Mark $=$ Int $\times$ Bool, so mark $::$ Int $\sim$ Mark.

The type of $\mathrm{map}_{T}$ is

$$
\operatorname{map}_{T} \quad:: \quad(a \sim b) \rightarrow(T a \leadsto T b)
$$

This function, whose definition will be given a little later on, is just like the ordinary map function associated with a datatype $T$ except that it can take a multifunction as argument and return a multifunction as result.

The combination map $_{T}$ mark denotes the operation of marking an element of $T$ Int in a completely nondeterministic way. The functional programmer may wonder at this point why multifunctions are being brought in. "Aren't we going to be interested in the set of all possible markings?", she may ask. The answer is: "Yes, but that will come later". It is notationally much simpler to consider things at the level of an arbitrary marking, and then move wholesale to the set level at the last 
possible moment. There is also another reason why multifunctions are necessary, as we will soon see.

Next, the function dom, which takes a multifunction as argument and returns a partial function as result, is defined as follows:

$$
\begin{array}{lll}
\text { dom } & :: & (a \leadsto b) \rightarrow(a \leadsto a) \\
\operatorname{dom} p & = & f s t \cdot\langle i d, p\rangle
\end{array}
$$

The split operation $\langle f, g\rangle$ is defined by $\langle f, g\rangle x=(f x, g x)$. The expression $\langle f, g\rangle$ denotes a multifunction that returns a result on an argument $x$ if and only if both $f$ and $g$ do. We are working with types as flat sets remember, and there is no $\perp$ or partially defined tuples. The function $f s t$ is standard and selects the first component of a (well-defined) pair. It will be appreciated from these remarks that dom $p$ applied to an argument $x$ returns $x$ if and only if $x$ is in the domain of $p$. Thus, $\operatorname{dom} p$ is a partial function included in the identity function. A partial function is a special case of a multifunction, namely a multifunction that returns either one value or none.

In the specification of $m m p$ the function $d o m$ is applied to a given multifunction test :: T Mark $\leadsto$ Test. For example, in the atiguous-marking problem, test = atig and Test $=$ Bool.

Next, the function value is defined as follows:

$$
\begin{array}{lll}
\text { value } & :: & \text { T Mark } \rightarrow \text { Int } \\
\text { value } & = & \text { sum } \cdot \text { map }_{T} \text { val } \\
\operatorname{val}(n, b) & = & \text { if } b \text { then } n \text { else } 0
\end{array}
$$

The subsidiary function sum :: T Int $\rightarrow$ Int for summing a structure of integers will be defined shortly.

Next, the operation $\Lambda$ turns a multifunction into the corresponding set-valued function:

$$
\begin{array}{lll}
\Lambda & :: & (a \leadsto b) \rightarrow(a \rightarrow \text { Set } b) \\
(\Lambda f) a & = & \{b \mid b \sim f a\}
\end{array}
$$

We write $b \backsim f a$ to denote the fact that $b$ is a possible value of $f a$. Thus $(\Lambda f) a$ returns the set of all possible values $b$ that can be returned as the result of applying the multifunction $f$ to $a$.

Finally, the multifunction max is defined by

$$
\begin{array}{ll}
\max & :: \quad(a \rightarrow a \rightarrow \text { Bool }) \rightarrow(\text { Set } a \leadsto a) \\
a \leftarrow \max (\unlhd) \text { as } & \equiv a \in \text { as } \wedge(\forall b \in \text { as }: b \unlhd a)
\end{array}
$$

In words, max takes an ordering $\unlhd$ and a set as as argument, and returns some maximum element in as under $\unlhd$. An ordering is also a relation, but it would seem strange to consider it as a multifunction, so we choose to represent it as a curried function returning a Boolean result. The minimum requirement on $\unlhd$ is that it should be a connected preorder, that is, a reflexive and transitive relation with the property that for all $x$ and $y$ either $x \unlhd y$ or $y \unlhd x$. (The usual name for a connected preorder is a total preorder but that name invites confusion because of 
the ambiguity of the word total.) Then we are guaranteed that max $(\unlhd)$ as produces at least one result for all nonempty sets as.

Note that $\max (\unlhd)$ as does not specify which element of as should be chosen. This freedom of action is crucial to the success of the reasoning to come, and is the second reason why the move to multifunctions is necessary.

\subsection{Folds and functors}

Two operations, sum and $\operatorname{map}_{T}$ were left unspecified above (the multifunction test is part of the input of the problem). To remedy the omission we need to say how $T$ is defined and what the fold function for $T$ is. In brief, a parameterised recursive datatype $T a$ can be defined as the least fixed point of another, so-called base datatype $F(a, b)$. The fixed-point property means that

$$
T a \cong F(a, T a)
$$

For example, List $a \cong 1+a \times$ List $a$, so List $a$ is a fixed point of $F(a, b)=1+a \times b$. The use of $\cong$ rather than equality is because the two types are isomorphic rather than identical. The constructors of a data declaration in Haskell are functions that convert the component types on the right-hand side into elements of the type being declared. We can parcel all constructors into just one function $i n_{T}: F(a, T a) \rightarrow$ $T a$. The converse function out $: T a \rightarrow F(a, T a)$, the other half of the isomorphism, is implicit in the permitted use of pattern matching with elements of declared datatypes.

The least fixed-point property of $T$ means that given any function $f:: F(a, b) \rightarrow$ $b$ we can construct a unique function $h:: T a \rightarrow b$ satisfying

$$
h \cdot i n_{T}=f \cdot \operatorname{map}_{F}(i d, h)
$$

The function $h$ is denoted by fold $F$. Thus fold $F::(F(a, b) \rightarrow b) \rightarrow(T a \rightarrow b)$. For example, sum $=$ fold $_{F}$ plus $_{F}$, where plus $_{F}:: F($ Int, Int $) \rightarrow$ Int. We cannot say what plus $_{F}$ is without knowing the structure of $F$. However, plus $F$ can be defined for the so-called regular functors $F$ (basically, polynomial functors closed under recursive data declarations) by induction over the structure of type constructors.

Given $f:: a \rightarrow x$ and $g:: b \rightarrow y$, the function $\operatorname{map}_{F}(f, g)$ has type $F(a, b) \rightarrow$ $F(x, y)$. Moreover, this function satisfies the two equations

$$
\begin{aligned}
\operatorname{map}_{F}(i d, i d) & =i d \\
\operatorname{map}_{F}(f \cdot h, g \cdot k) & =\operatorname{map}_{F}(f, g) \cdot \operatorname{map}_{F}(h, k)
\end{aligned}
$$

In a word, $F$ is a functor. We will make free use of the above equations in what follows without always making it explicit that we are doing so.

Given $f:: a \rightarrow b$, the combination $i n_{T} \cdot \operatorname{map}_{F}(f, i d)$ has type $F(a, T b) \rightarrow T b$; consequently fold $f_{F}\left(i n_{T} \cdot \operatorname{map}_{F}(f, i d)\right):: T a \rightarrow T b$. As might be suggested by this type signature, we have

$$
\operatorname{map}_{T} f=\operatorname{fold}_{F}\left(i n_{T} \cdot \operatorname{map}_{F}(f, i d)\right)
$$


Thus the action of $T$ on functions is defined. Moreover, one can show that

$$
m a p_{T} i d=i d \text { and } \operatorname{map}_{T}(f \cdot g)=\operatorname{map}_{T} f \cdot m a p_{T} g
$$

Hence $T$ is also a functor. The proof of this claim involves two facts that will be useful later on. The identity law states that $f_{o l d} i n_{T}$ is the identity function on T a. Thus,

$$
\operatorname{map}_{T} i d=\operatorname{fold}_{F}\left(i n_{T} \cdot \operatorname{map}_{F}(i d, i d)\right)=\operatorname{fold}_{F} i n_{T}=i d
$$

and the first part is proved. The second law is called type-functor fusion and states that

$$
\operatorname{fold}_{F} f \cdot \operatorname{map}_{T} g=\operatorname{fold}_{F}\left(f \cdot \operatorname{map}_{F}(g, i d)\right)
$$

In words, a fold after a map can always be re-expressed as a single fold. We omit the simple proof that this gives the second claim.

All the above extends to the case that the argument of a fold is a multifunction rather than a plain function. There are one or two details that have to be addressed in a full explanation (such as what precisely does the functor $\times$ mean in a relational setting), but we will not go into them. It suffices to state that we can take

$$
\text { fold }_{F} \quad:: \quad(F(a, b) \leadsto b) \rightarrow(T a \leadsto b)
$$

In particular, we can replace both occurrences of $\sim$ by $\rightarrow$ in this type signature.

\subsection{The banana-split and fusion laws}

We need two other pieces of technical machinery before we can proceed with deriving an implementation of $m m p$. The first is called the banana-split law, and states that

$$
\left\langle\operatorname{fold}_{F} f, \text { fold }_{F} g\right\rangle=\operatorname{fold}_{F}\left\langle f \cdot \operatorname{map}_{F}(i d, f s t), g \cdot \operatorname{map}_{F}(i d, \text { snd })\right\rangle
$$

In words, a split involving two folds can be rewritten as a fold involving a split. (In the old days, folds were written with "banana" brackets, hence the catchy name.)

The second piece of machinery is the fusion condition

$$
f \cdot \text { fold }_{F} g \supseteq \text { fold }_{F} h \Leftarrow f \cdot g \supseteq h \cdot \operatorname{map}_{F}(i d, f)
$$

The fusion condition also holds when both occurrences of $\supseteq$ are replaced by $=$, or replaced by $\subseteq$. See (Bird,1998) for a discussion of the fundamental role of fusion in proving facts about functional programs. In fact, type-functor fusion is a special case of fusion. We will need fusion in Section 5.

\section{Rewriting the specification}

To save looking back, here is the specification of $m m p$ again:

$$
m m p=\max (\leq) \cdot \Lambda\left(\text { value } \cdot \text { dom test } \cdot \text { map }_{T} \text { mark }\right)
$$

Let us start with the subexpression value $\cdot$ dom test:

value $\cdot$ dom test 


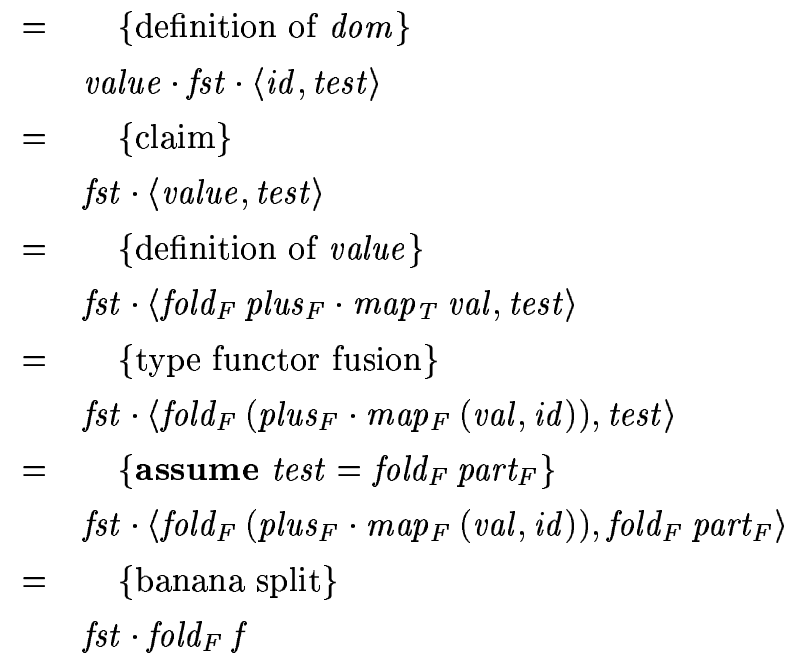

where, setting Result $=$ Int $\times$ Test, we have

$$
\begin{aligned}
& f:: \quad F(\text { Mark }, \text { Result }) \leadsto \text { Result } \\
& f=\left\langle\text { plus }_{F} \cdot \operatorname{map}_{F}(\text { val }, f s t), \text { part }_{F} \cdot \operatorname{map}_{F}(i d, \text { snd })\right\rangle
\end{aligned}
$$

The claim is a consequence of two laws:

$$
\begin{array}{ll}
f \cdot f s t & =f s t \cdot(f \times i d) \\
(f \times g) \cdot\langle h, k\rangle & =\langle f \cdot h, g \cdot k\rangle
\end{array}
$$

where $(f \times g)(x, y)=(f x, g y)$. Note our assumption on test :: T Mark $\rightarrow$ Test, namely that test $=$ fold $_{F}$ part $_{F}$, where part $_{F}:: F($ Mark, Test $) \leadsto$ Test.

Now we can continue:

$$
\begin{aligned}
& \text { value } \cdot \text { dom test } \cdot \text { map }_{T} \text { mark } \\
& =\{\text { above }\} \\
& f_{s t} \cdot \text { fold }_{F} f \cdot \text { map }_{T} \text { mark } \\
& =\quad \text { type functor fusion }\} \\
& \text { fst } \cdot \text { fold }_{F} g
\end{aligned}
$$

where $g:: F($ Int, Result $) \sim$ Result is defined by $g=f \cdot m^{2} p_{F}($ mark, $i d)$. Using this result, we now obtain

$$
\begin{aligned}
& \max (\leq) \cdot \Lambda\left(\text { value } \cdot \text { dom } p \cdot \text { map }_{T} \text { mark }\right) \\
= & \quad\{\text { above }\} \\
& \max (\leq) \cdot \Lambda\left(\text { fst } \cdot \text { fold }_{F} g\right) \\
= & \{\text { claim }\} \\
& f s t \cdot \max \left(\leq_{1}\right) \cdot \Lambda\left(\text { fold }_{F} g\right)
\end{aligned}
$$

where $\left(\leq_{1}\right)::$ Result $\rightarrow$ Result $\rightarrow$ Bool is defined by

$$
\left(a_{1}, b_{1}\right) \leq_{1}\left(a_{2}, b_{2}\right) \hat{=}\left(a_{1} \leq a_{2}\right)
$$


The claim is intuitively obvious but to justify it calculationally would involve more notation than we want to expose. See (Bird and de Moor,1996) for the proof.

Where are we? Well, we have in effect reduced the MMP problem to one of computing an expression of the form $\max (\unlhd) \cdot \Lambda\left(\right.$ fold $\left._{F} h\right)$ efficiently. The only assumption was that test can be expressed as a fold. Time now for some standard theory.

\section{Thinning}

How can we compute an expression $\max (\unlhd) \cdot \Lambda\left(\right.$ fold $\left._{F} h\right)$, given suitable definitions of $\unlhd$ and $h$ ? One possibility is to make use of the Eilenberg-Wright theorem, which says that

$$
\Lambda\left(\text { fold }_{F} h\right)=\operatorname{fold}_{F}\left(\Lambda\left(h \cdot \operatorname{map}_{F}(i d, \text { choose })\right)\right)
$$

where choose :: Set $a \leadsto a$ is the membership relation for sets (so $a \backsim$ choose as iff $a \in a s$ ). In words, the set of results returned by a relational fold can be obtained as a functional fold that at each stage returns the set of all possible intermediate results. Proof of the Eilenberg-Wright theorem is given in (Bird and de Moor,1996), as are the proofs of other results in this section.

At the other extreme lies the Greedy theorem, which states that

$$
\max (\unlhd) \cdot \Lambda\left(\text { fold }_{F} h\right) \supseteq \operatorname{fold}_{F}(\max (\unlhd) \cdot \Lambda h)
$$

provided $h:: F(a, b) \leadsto b$ is monotonic under the preorder $(\unlhd):: b \rightarrow b \rightarrow$ Bool, that is,

$$
x \unlhd_{F} y \wedge u \backsim h x \Rightarrow(\exists v: v \backsim h y \wedge u \unlhd v)
$$

where $\left(\unlhd_{F}\right):: F(a, b) \rightarrow F(a, b) \rightarrow$ Bool is the ordering on $F$ induced by $\unlhd$. Note that the Greedy theorem asserts a refinement relation between the two sides, not an equality. In words, the Greedy theorem says that some optimum result (not necessarily every optimal result) can be computed by maintaining a single optimum partial result at each stage of the folding process.

For the MMP problem, $h:: F$ (Int, Result) $\leadsto$ Result is given by

$$
h=\left\langle\text { plus }_{F} \cdot \operatorname{map}_{F}(\text { val }, f s t), \operatorname{part}_{F} \cdot \operatorname{map}_{F}(i d, s n d)\right\rangle \cdot \operatorname{map}_{F}(\text { mark, id })
$$

and it is not too difficult to see that $h$ is not monotonic under $\leq_{1}$ : we can have $x \leq_{1 F} y$ and $h x$ returning some result without having $h y$ returning any result because $\leq_{1}$ does not depend on second components. Hence monotonicity fails.

The minimum generalisation that restores monotonicity is to define $\leq_{2}$ by

$$
\left(a_{1}, b_{1}\right) \leq_{2}\left(a_{2}, b_{2}\right) \hat{=}\left(a_{1} \leq a_{2} \wedge b_{1}=b_{2}\right)
$$

Then it is fairly easy to see that $h$ is monotonic under $\leq_{2}$. The problem is that $\leq_{2}$ is not a connected preorder, so we cannot expect $\max \left(\leq_{2}\right)$ as to return a value for all nonempty sets as.

What saves the day is the idea of thinning. Define

$$
\begin{array}{lll}
\text { thin } & :: \quad(a \rightarrow a \rightarrow B o o l) \rightarrow(\text { Set } a \leadsto \text { Set } a) \\
b s \leftarrow \text { thin }(\preceq) \text { as } & \equiv \quad b s \subseteq \text { as } \wedge(\forall a \in \text { as }: \exists b \in b s: a \preceq b)
\end{array}
$$


In words, thin takes a not necessarily connected preorder $\preceq$ and a set as and nondeterministically returns some subset $b s$ of $a s$ with the property that all elements of as have an upper bound under $\preceq$ in $b s$.

The thinning theorem states that

$$
\max (\unlhd) \cdot \Lambda\left(\text { fold }_{F} h\right) \supseteq \max (\unlhd) \cdot \text { fold }_{F} k
$$

where

$$
\begin{aligned}
& k:: \quad F(a, \text { Set } b) \leadsto \text { Set } b \\
& k=\operatorname{thin}(\preceq) \cdot \Lambda\left(h \cdot \operatorname{map}_{F}(i d, \text { choose })\right)
\end{aligned}
$$

provided that: (i) $x \preceq y \Rightarrow x \unlhd y$; and (ii) $h$ is monotonic under $\preceq$. In words, the thinning theorem says that we can compute an optimum result by maintaining a representative number of partial solutions at each stage of the folding process.

How many partial solutions have to be kept on the go for the MMP? Look again at the definition of $\leq_{2}$ (the instantiation for $\preceq$ ) and observe that the second components $b_{1}$ and $b_{2}$ are each elements of Test, the target type of test. If Test has size $k$, then we need keep at most $k$ partial solutions at each stage. For the atiguous problem, Test $=$ Bool, so only two partial solutions have to be kept. As we will see later on, Test will also have finite size for the other problem described in the introduction.

\section{Implementation}

As a consequence of applying the thinning theorem to the MMP problem we have

$$
\begin{aligned}
& m m p \supseteq f_{s t} \cdot \max \left(\leq_{1}\right) \cdot \text { fold }_{F} k \\
& k=\operatorname{thin}\left(\leq_{2}\right) \cdot \Lambda\left(f \cdot \operatorname{map}_{F}(\text { mark, choose })\right) \\
& f \quad=\left\langle\operatorname{plus}_{F} \cdot \operatorname{map}_{F}(\text { val, fst }), \text { part }_{F} \cdot \operatorname{map}_{F}(i d, \text { snd })\right\rangle
\end{aligned}
$$

The types of these multifunctions are as follows:

$$
\begin{array}{lll}
m m p & :: & \text { T Int } \rightarrow \text { Int } \\
k & :: & F(\text { Int }, \text { Set Result }) \sim \text { Set Result } \\
f & :: & F(\text { Mark, Result }) \leadsto \text { Result }
\end{array}
$$

Our task now is to implement the various functions in Haskell. In particular, fold $F$ can be implemented as in Figure 3 which uses Haskell's newtype construction to introduce the type $T$. The base functor $F$ has to be supplied to complete the definition. Functions not decorated with an $F$ subscript can be implemented independently of the details of any particular marking problem.

The aim of the game is to represent sets by lists and set-processing functions by list-processing ones. In particular, the functions maxlist $::$ List $a \rightarrow a$ and thinlist :: List $a \rightarrow$ List $a$ are specified by

$$
\begin{array}{lll}
\max (\unlhd) & \supseteq & \text { maxlist }(\unlhd) \cdot \text { listify } \\
\operatorname{thin}(\unlhd) & \supseteq & \text { setify } \cdot \text { thinlist }(\unlhd) \cdot \text { listify }
\end{array}
$$

where setify $::$ List $a \rightarrow$ Set a converts a list into the set of its elements and listify :: 


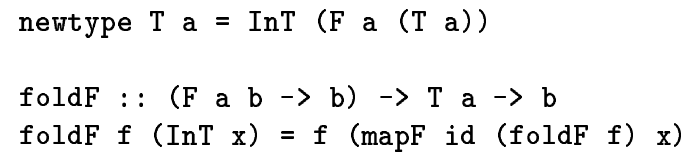

Fig. 3. The datatype $T a$

Set $a \sim$ List $a$ does the reverse. Thus

$$
\text { setify } \cdot \text { listify }=i d \quad \text { and } \quad \text { listify } \cdot \text { setify } \supseteq \text { id }
$$

Use of $\supseteq$ rather than $=$ is necessary in these formulae because we are replacing multifunctions by functions and hence exorcising nondeterminism. Figure 4 gives possible implementations of maxlist and thinlist.

It is important to note that thinlist works by comparing every element on the list with every other, and so takes quadratic time in the length of the list. Although thinlist is optimal at thinning, its quadratic behaviour is not acceptable for thinning algorithms in general. A better solution, explored in (Bird and de Moor,1996), is to implement a linear-time version of thinlist and combine it with a implementation of sets as sorted lists that bring candidates for thinning together, so allowing the linear-time version to be effective at thinning.

However, for an MMP problem that maintains $k$ partial solutions at each step, thinlist will be applied to a list of length at most $2 k$ at each step because application of mark doubles the number of candidates. Hence the quadratic definition of thinlist is acceptable, indeed welcome because it implies that we can implement a set by listing its elements in any order we like.

We have to implement $\Lambda$ as a list-generating function. As a first step we "localize" occurrences of $\Lambda$ by making use of three rules:

$$
\begin{aligned}
& \Lambda(f \cdot g)=\text { union } \cdot \operatorname{map}_{S}(\Lambda f) \cdot \Lambda g \\
& \Lambda\langle f, g\rangle=c p \cdot\langle\Lambda f, \Lambda g\rangle \\
& \Lambda\left(\operatorname{map}_{F}(f, g)\right)=c p_{F} \cdot \operatorname{map}_{F}(\Lambda f, \Lambda g)
\end{aligned}
$$

The subsidiary functions have types

$$
\begin{array}{lll}
\text { union } & :: & \text { Set }(\text { Set } a) \rightarrow \text { Set } a \\
\text { map }_{S} & :: & (a \rightarrow b) \rightarrow(\text { Set } a \rightarrow \text { Set } b) \\
c p & :: & \text { Set } a \times \text { Set } b \rightarrow \text { Set }(a \times b) \\
c p_{F} & :: & F(\text { Set } a, \text { Set } b) \rightarrow \operatorname{Set}(F(a, b))
\end{array}
$$

The function union takes the union of a set of sets, map $_{S}$ is the map function for sets, $c p$ takes the cartesian product of two sets, and $c p_{F}$ is the generalisation of $c p=c p_{\times}$to an arbitrary $F$.

If $g:: a \rightarrow b$, then $\Lambda g=\tau \cdot g$, where $\tau:: b \rightarrow$ Set $b$ returns a singleton set. In such a case we have

$$
\Lambda(f \cdot g)=\Lambda f \cdot g
$$

Using these rules, together with the fact that $\Lambda$ choose is the identity function on 


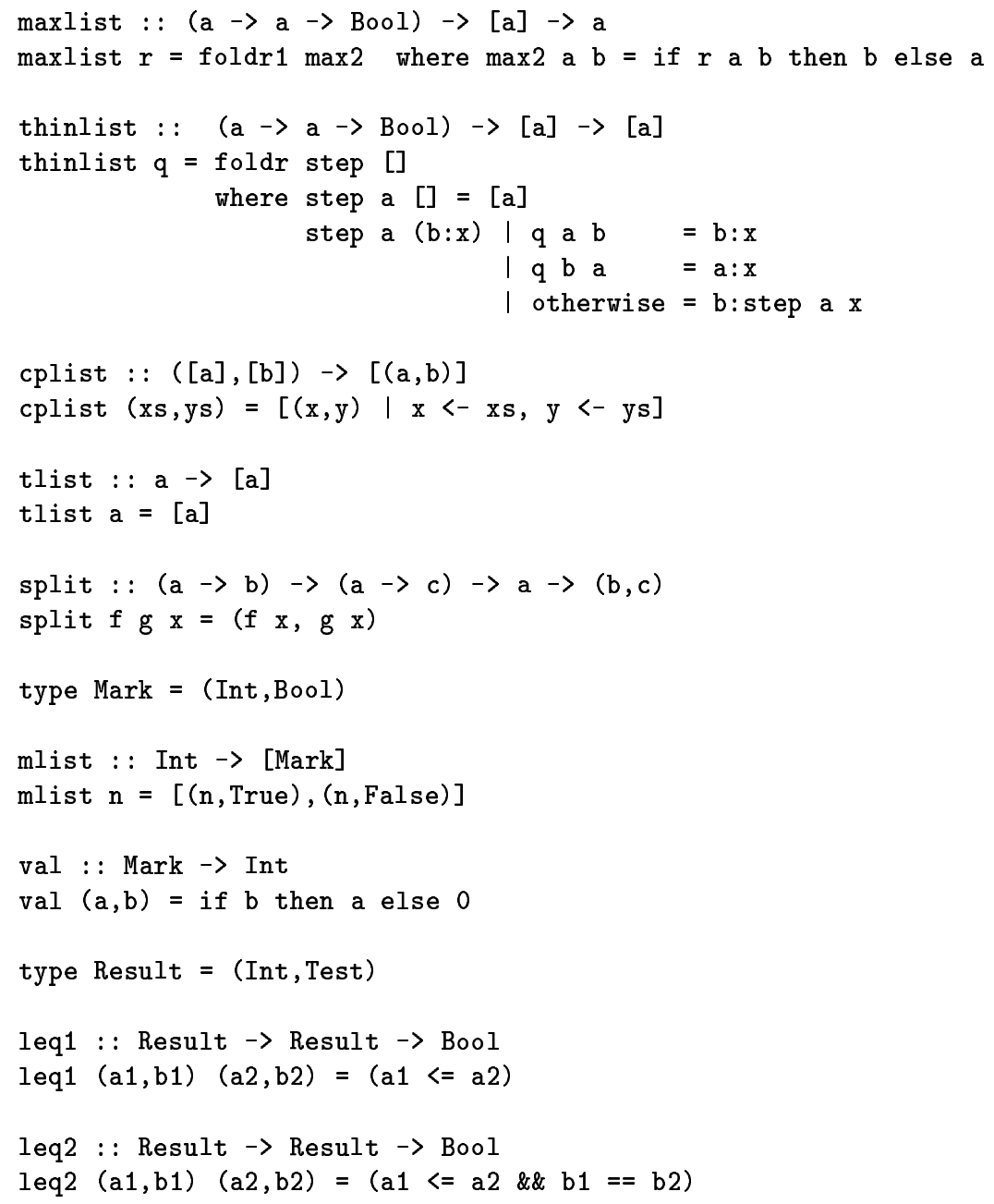

Fig. 4. Generic utility functions

sets, we can rewrite the specification of $m m p$ to read:

$$
\begin{aligned}
& m m p \supseteq f s t \cdot \max \left(\leq_{1}\right) \cdot \operatorname{fold}_{F} k \\
& \left.k=\text { thin }\left(\leq_{2}\right) \cdot \text { union } \cdot \operatorname{map}_{S} f \cdot c p_{F} \cdot \operatorname{map}_{F}(\Lambda \operatorname{mark}, i d)\right) \\
& f \quad=c p \cdot\left\langle\tau \cdot \text { plus }_{F} \cdot \operatorname{map}_{F}(\text { val }, f s t), \Lambda \operatorname{part}_{F} \cdot \operatorname{map}_{F}(i d, \text { snd })\right\rangle
\end{aligned}
$$

The type of $f$ is now $f:: F($ Mark, Result $) \rightarrow$ Set Result.

Let us now implement $f$. We will need three functions cplist, tlist and plist $F$ satisfying

$$
\begin{aligned}
& \text { cp } \cdot(\text { setify } \times \text { setify })=\text { setify } \cdot \text { cplist } \\
& \tau \quad=\text { setify } \cdot \text { tlist } \\
& \Lambda_{\text {part }_{F}}=\text { setify } \cdot \text { plist }_{F}
\end{aligned}
$$




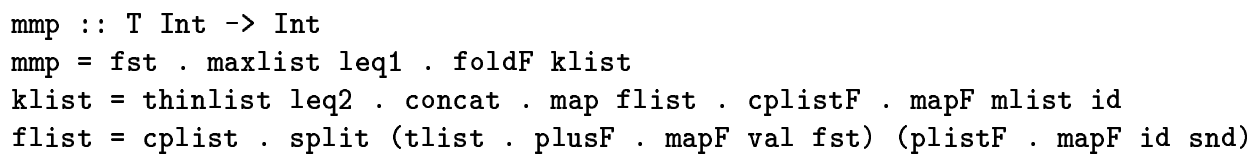

Fig. 5. The generic program

Definitions of $c p l i s t$ and tlist are given in Figure 4; the definition of plist $_{F}$ depends on the particular marking problem and will be given later. Installing these definitions we obtain $f=$ setify . flist, where flist is given in Figure 5.

Turning to $k$, we will need functions mlist and cplist $_{F}$ satisfying

$$
\begin{array}{ll}
\Lambda \operatorname{mark} & =\text { setify } \cdot \text { mlist } \\
c p_{F} \cdot \operatorname{map}_{F}(\text { setify, setify }) & =\text { setify } \cdot \text { cplist }_{F}
\end{array}
$$

Figure 4 gives one implementation of mlist; the definition of cplist $_{F}$ depends on $F$ and will be given later. Installing these identities and using the rules relating listify and setify given above, together with the equation

$$
\text { union } \cdot \text { map }_{S} \text { setify } \cdot \text { setify }=\text { setify } \cdot \text { concat }
$$

we obtain

$$
\text { listify } \cdot k \quad \supseteq \quad k \text { list } \cdot \operatorname{map}_{F}(\text { id, listify })
$$

where klist is defined in Figure 5.

Finally, we turn to $m m p$ and reason:

$$
\begin{array}{lc} 
& m m p \\
\supseteq & \{\text { given }\} \\
& f s t \cdot \max \left(\leq_{1}\right) \cdot \text { fold }_{F} k \\
\supseteq & \{\text { specification of } \text { maxlist }\} \\
& \text { fst } \cdot \text { maxlist }\left(\leq_{1}\right) \cdot \text { listify }^{\prime} \text { fold }_{F} k \\
\supseteq & \{\text { fusion } ; \text { see above }\} \\
& f s t \cdot \text { maxlist }\left(\leq_{1}\right) \cdot \text { fold }_{F} \text { klist }
\end{array}
$$

The result as a Haskell program is summarised in Figure 5.

\section{Applications}

Finally we are in a position to instantiate the generic marking problem for our two examples. Figure 6 gives the instantiations of the remaining functions $\operatorname{map}_{F}$, cplist $_{F}$, plus $_{F}$ and plist $_{F}$ for the atiguous problem on binary trees.

In order to bring out a crucial point about efficiency, we have declared the type of cplist $_{F}$ to be the instance at which it is used in the generic marking problem. If Result has size $k$ (and $k=2$ in the atiguous problem), then the thinning algorithm maintains $k$ partial results at each step. Consequently, cplist $_{F}$ generates $2 k^{2}$ 


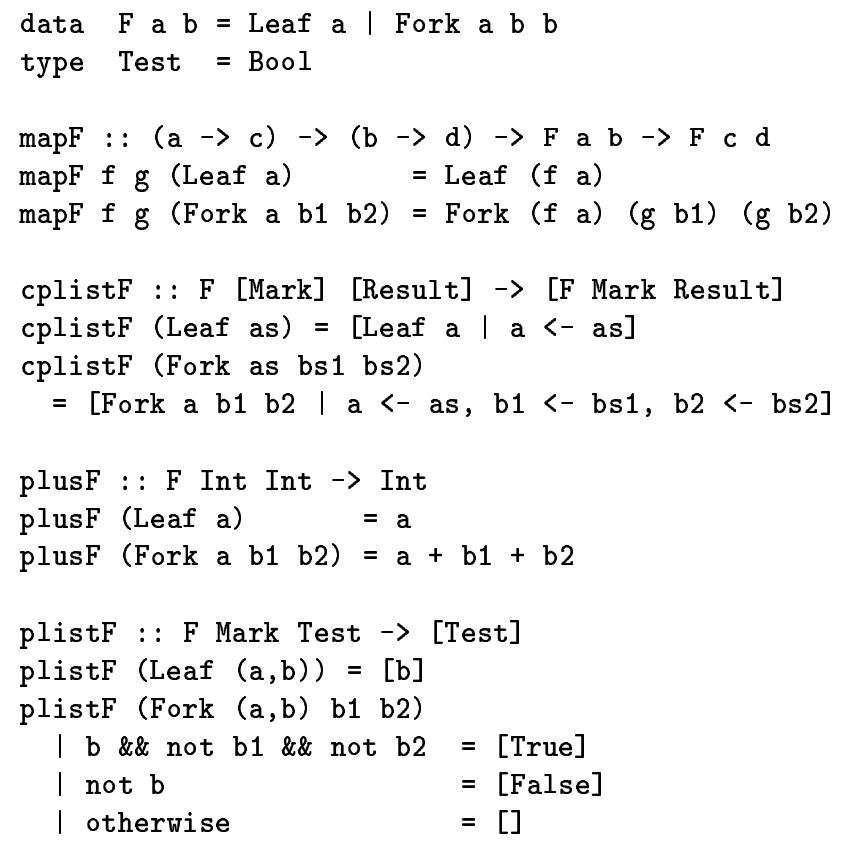

Fig. 6. The atiguous problem

candidate new partial results for subsequent thinning. These partial results can be processed in constant time, so the complete algorithm requires linear time.

The situation changes when the base functor $F$ is not polynomial, as is the case with rose trees. For rose trees we have $F(a, b)=a \times$ List $b$, and for a node with $n$ immediate offspring, the associated function cplist $_{F}$ will produce a list of $2 k^{n}$ candidates. Although these candidates are subsequently thinned to only $k$ results, the process will no longer take constant time. In fact, direct instantiation of any non-trivial marking problem for rose trees will take exponential time.

The only way out of this unfortunate situation seems to be to recast a marking problem for rose trees as a marking problem for leaf-labelled binary trees, exploiting the isomorphism between rose trees and binary trees illustrated in Figure 7. This is also the resolution proposed by (Sasano et al.,2000) though the problem is not identified in quite the same way.

Figure 8 gives the instantiation for the contiguous problem for rose trees, expressed as a problem on binary trees. The type Test contains three values: $A$ signifies that the tree is contiguous and the root is marked; $B$ that the tree is contiguous and the root is unmarked (that is, marked False), and $C$ that the tree is completely unmarked. The definition of plist $_{F}$ is reasonably clear once one ploughs through the clauses. 


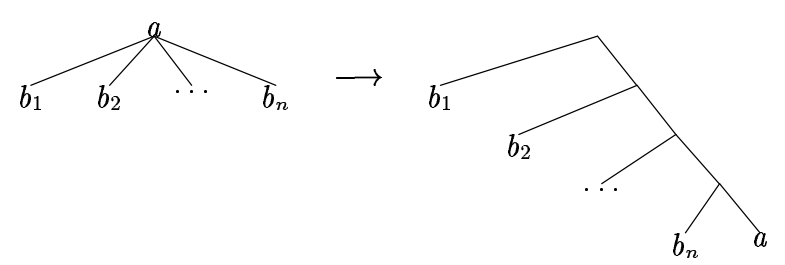

Fig. 7. Isomorphism between rose trees and leaf-labelled binary trees

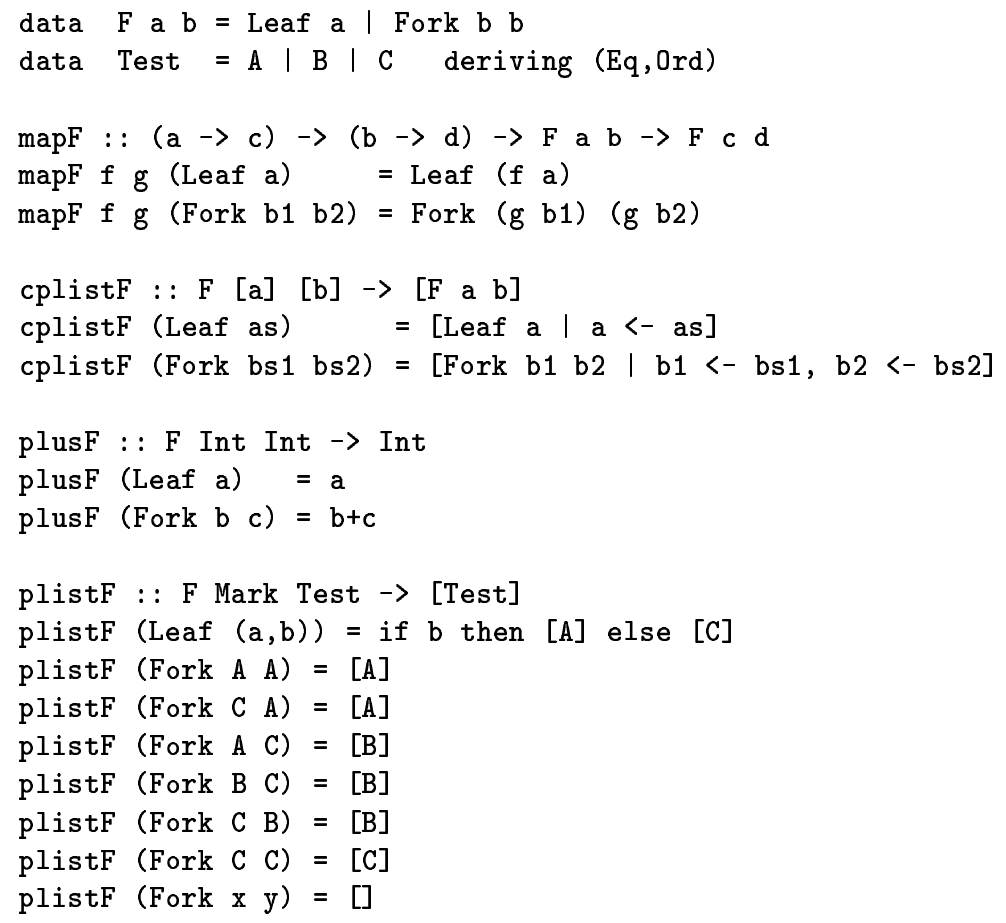

Fig. 8. The contiguous problem

\section{Conclusions}

Consider the benefits of the treatment above: we have derived a generic solution to all marking problems whose constraint can be expressed as a fold involving a multifunction. The only fly in the ointment is that, for efficiency, any problem involving a datatype that is not based on a polynomial functor has to be re-expressed in terms of a datatype that is. What is more, we have shown how thinning algorithms can solve a whole range of optimisation problems. (Oh, yes, the two puzzles yield maximum values 28 and 18 respectively). 


\section{References}

Bird, R. (1998) Introduction to Functional Programming using Haskell, Prentice Hall International.

Bird, R. and de Moor, O. (1998) The Algebra of Programming, Prentice Hall International. Sasano, I, Hu, Z, Takeichi, M, and Ogawa, M. (2000) Calculating linear-time algorithms for solving maximum weightsum problems. Proc International Conference on Functional Programming, Montreal, Canada. 